nau wie die anderen vom Bundesamt für Sicherheit in der Informationstechnik zugelassenen SINA Workstation Arbeitsplätze, alle Vorteile der Multilevel- und Multisession-Funktionalität und lässt sich leicht in bestehende Infrastrukturen integrieren. Darüber hinaus stellt das System spezifische SINA Applikationen bereit, die zum Beispiel gesicherte IP-Telefonie oder Video-Konferenzen mit Microsoft Lync ermöglichen.

\section{DSRI Ausschreibung: Förderpreise 2014}

Die Deutsche Stiftung für Recht und Informatik (DSRI) verleiht auch 2014 wieder Förderpreise in zwei Kategorien:

\section{DSRI-Wissenschaftspreis}

für eine herausragende Dissertation oder Habilitationsschrift auf dem Gebiet des Informationsrechts oder der Rechtsinformatik, dotiert mit $2.000 €$.

\section{DSRI-Absolventenpreis}

für eine herausragende:

- Diplom- oder Magisterarbeit,

- Bachelor- oder Master-Thesis

- oder sonstige Abschlussarbeit, dotiert mit $500 €$.

- Die Arbeit soll

- eine besondere Exzellenz aufweisen,

- zum Zeitpunkt der Einreichung seit max. 2 Jahren abgeschlossen oder publiziert worden sein und

- von einer betreuenden Lehrperson vorgeschlagen werden, die die Einreichung der Arbeit begründet.

Die Preise werden jährlich vergeben. Das Preisgeld wird von der DGRI zur Verfügung gestellt. Die nächste Preisverleihung erfolgt auf der DGRI-Jahrestagung im November 2014. Einreichungsschluss ist der 6 . Juni 2014 bei u. g. Anschrift. Die Vorschläge werden von der vom Stiftungsrat der DSRI eingesetzten Kommission bewertet.

Die Bewerber/innen sollen von einer betreuenden Lehrperson vorgeschlagen werden, die begründet, weshalb die eingereichte Arbeit eine besondere Exzellenz aufweist. Die Arbeit sollte nicht vor 2012 abgeschlossen oder publiziert worden sein. Mit Annahme des Preises ist die Überlassung des gedruckten Werkes an die DSRI verbunden.

Einreichungen sind zu richten an:

Deutsche Stiftung für Recht und Informatik (DSRI)

Kurwickstr. 14 - 15, 26122 Oldenburg

E-Mail:info@dsri.de

\section{a-i3/BSI Symposium 2014}

Die Arbeitsgruppe Identitätsschutz im Internet (a-i3) und das Bundesamt für Sicherheit in der Informationstechnik (BSI) laden ein zum 9. interdisziplinären Symposium:

Sicherheit von Daten und Identitäten angesichts NSA und BigData, am 19 und 20. Mai 2014 an der Ruhr-Universität Bochum.

Vertreter aus Unternehmen, Wissenschaft, Politik und Verbänden diskutieren unter dem diesjährigen Oberthema „Sicherheit von Daten und Identitäten angesichts NSA und Big Data" aktuelle Fragen, die sich im Zusammenhang mit den Erkenntnissen über Spionage und den aktuellen Entwicklungen der Datenverarbeitung ergeben.

Gegenstand der Tagung sind Aspekte der Sicherheit von Daten und Identitäten in privater und industrieller Datenverarbeitung, die insbesondere aus technischer und rechtlicher Sicht beleuchtet werden. Einen Schwerpunkt bildet das Thema "Spionage und Cybercrime", wobei die Überwachung durch Geheimdienste und aktuelle Angriffe auf Identitäten im Mittelpunkt stehen. Hier stellt sich die Frage, ob und wie sich Unternehmen und Private vor Informationsspionage und Identitätsdiebstahl schützen können.

Einen weiteren Schwerpunkt bilden Standards für die Cloud und die aktuell diskutierte Zertifizierung von Cloud-Diensten. Hier wird es darum gehen, aktuelle Standards für Cloud-Dienste zu beleuchten, etwa den offenen OASIS-Standard oder die sich im Entwurfsstadium befindliche ISO-Norm 27018. Daneben sollen Zertifizierungsmodelle aufgezeigt werden, die eine (rechts-)sichere Auslagerung von IT-Prozessen in die Cloud ermöglichen. Eine besondere Bedeutung kommt auch der Frage zu, wie Privatheit im Kontext von Cloud und Big Data gewährleistet werden kann und wie sich technische Möglichkeiten, beispielsweise Verschlüsselungstechniken, hierauf auswirken.

Ebenfalls auf dem Programm steht das Thema „Identifizierung und Identitätsschutz". Die kommende, europaweit geltende EIdentity-Verordnung wird neue Regelungen zur Identifizierung in weiten Teilen des Internets schaffen. Darüber hinaus werden Fragen der Behördenkommunikation thematisiert: Das in Kraft getretene E-Government-Gesetz und die fortschreitende elektronische Kommunikation in der Justiz werfen zahlreiche rechtliche Problemstellungen auf. Zuletzt sollen hier auch Angriffe auf Single-SignOn-Plattformen dargestellt werden.

Schließlich wird der Themenkomplex um Big Data und industrielle Datenverarbeitung in den Blick genommen: Zentrales Element ist hier das Identitätsmanagement, das eine sichere Identifizierung gewährleisten soll. Neben der technischen Realisierung sind auch datenschutzrechtliche Aspekte zu beachten. Dabei sollen Fragen zum betrieblichen Datenschutz, insbesondere bei Unternehmenskooperationen, geklärt werden. Die anschließende Podiumsdiskussion zum Thema „Identität und Persönlichkeit im Zeitalter von Big Data" wird Anlass geben, das Spannungsfeld zwischen Persönlichkeitsschutz und Wirtschaftsinteressen zu diskutieren.

Die Veranstaltung richtet sich an Entscheidungsträger von Verwaltungsbehörden; an Leiter, Mitarbeiter und Datenschutzbeauftragte in Organisationen und Unternehmen aus den Gebieten ITSicherheit, Softwareentwicklung und E-Commerce; weiterhin an Juristen in Justiz, Unternehmen und Verbänden; spezialisierte Rechtsanwälte sowie Leiter und Mitarbeiter in Aufsichts- und Datenschutzbehörden.

Das Anmeldeformular zum Symposium finden Sie hier: http:// www.ruhr-uni-bochum.de/Is-borges/sympo/a-i3_BSI-Symposium 2014_Anmeldung.pdf

\section{FhG-SIT: Lagebericht zum Web-Tracking}

Das Fraunhofer-Institut für Sichere Informationstechnologie in Darmstadt hat am 12.03.2014 den Web-Tracking-Report 2014 veröffentlicht. Über ein Jahr haben Mitarbeiter des Instituts regelmäBig mehr als 1600 von Deutschlands beliebtesten Internet-Auftritten analysiert und geprüft, in welchem Umfang dort das Nutzerverhalten durch Web-Tracking erfasst wird: Oft sind 50 verschiede- 